\title{
Influence of On-Site Coulomb Interaction on Parameters of Superconducting State in Two-Dimensional Lattices
}

\author{
M.M. AdAMCZYK ${ }^{a, *}$, K.A. KROK ${ }^{a}$, \\ K.P. KOSK-JONIEC ${ }^{a}$ AND R. SzCZĘŚNIAK ${ }^{a, b}$ \\ ${ }^{a}$ Department of Theoretical Physics, Jan Długosz University in Częstochowa, \\ al. Armii Krajowej 13/15, 42-200 Częstochowa, Poland \\ ${ }^{b}$ Institute of Physics, Częstochowa University of Technology, \\ al. Armii Krajowej 19, 42-200 Częstochowa, Poland \\ Doi: 10.12693/APhysPolA.139.578 *e-mail: marta.adamczyk@doktorant.ujd.edu.pl
}

\begin{abstract}
The study examined the effect of electron correlations characterized by the on-site Coulomb integral $(U)$ on the parameters of the superconducting state in two-dimensional model lattices: square and triangular. In the electronic dispersion relation, we included the $U$ parameter using the Zubarev-type thermodynamic Green functions formalism. We determined the Eliashberg function $\left(\alpha^{2} F(\omega)\right)$ using standard analytical formulas. In particular, we examined the course of the electron density of state function $(\rho(\varepsilon))$ and $\alpha^{2} F(\omega)$ depending on $U$. Having the explicit Eliashberg functions, derived for specific values of the $U$ parameter, we analyzed the influence of on-site electron correlations on the parameters of the superconducting state: the electron-phonon coupling constant $(\lambda)$ and critical temperature $\left(T_{c}\right)$. We obtained completely different results between the two systems under consideration: in a square lattice, for the increasing Coulomb repulsion force, the superconducting state disappears. In a triangular lattice, there is a range of $U$ values for which the superconducting state is amplified. This is due to the decreasing (increasing) density of states at the Fermi level with the increase of the $U$ parameter for a square (triangular) lattice.
\end{abstract}

topics: on-site Coulomb interaction, superconducting parameters, two-dimensional lattice

\section{Introduction}

The influence of electron correlations on the superconducting state has not yet been thoroughly investigated in the literature. The reason for this is the extreme difficulty of considering the repulsive electron force in the superconducting state theory. Although the derivation of the equations describing the phonon-induced superconducting state together with the on-site electron interaction is complicated but feasible, solving these equations in a self-consistent manner is not easy.

Therefore, in the superconducting state theory (in the Eliashberg formalism) [1, 2], the depairing electron correlations are parametrically modeled by introducing the Coulomb pseudopotential $\left(\mu^{\star}\right)[3-5]$. The Coulomb parameter in the analyzed approach plays the role of a fitting parameter rather than a real quantity determining the actual Coulomb repulsion, which can be modeled with an on-site Hubbard integral $(U)$. This fact is related to the complicated definition of $\mu^{\star}$, which includes parameters such as: $U$, the density of state at the Fermi level $(\rho(0))$, the characteristic electron $\left(\omega_{e}\right)$ and the phononic $\left(\omega_{\mathrm{ph}}\right)$ frequency. Additionally, in the quantitative Eliashberg theory $[1,2]$, it enters the equation for the order parameter with the cutoff frequency $\omega_{\text {cut }}$, the value of which is very difficult to determine [6]. Accordingly, we are able to describe correctly phonon-induced superconductivity without electron correlations, but still we have little understanding of the significance and effect electronelectron interactions on the superconducting state.

The Eliashberg formalism has two input parameters: the Eliashberg function $\left(\alpha^{2} F(\omega)\right)$, which in its standard form models the electron-phonon interaction [7], and $\mu^{\star}$. The aim of the study is to derive the Eliashberg function for the model systems under consideration, the courses of which also take into account the Coulomb repulsion force in an effective manner. This will allow for the elimination of the Coulomb pseudopotential as an input parameter to the model and its complicated definition. The results of this work can be used as an example of modeling depairing electron correlations in the Eliashberg formalism, without using the Coulomb pseudopotential. Besides, having explicit 
data of Eliashberg functions that take into account the on-side electron interaction, one can easily, in an analytical way, estimate the basic parameters of the superconducting state, such as: the electron-phonon coupling constant $(\lambda)$ and the critical temperature $T_{c}$.

\section{Description of results}

The study of two-dimensional model crystal lattices in this context seems very interesting due to the van Hove singularity states occurring in the electron density. The existence of peaks near the Fermi level $(\rho(0))$ (see functions presented in Fig. 1 with solid black lines) may have a significant impact on the analyzed parameters of the superconducting state. In the first step of our analysis, it was necessary to determine the electronic dispersion relation for the two-dimensional lattices considered in the work. We used the tight binding method [8], obtaining for a square lattice (SQ):

$$
\begin{gathered}
\varepsilon_{\boldsymbol{k}}=-2 t\left[\cos \left(k_{x}\right)+\cos \left(k_{y}\right)\right] \\
+4 t^{\prime} \cos \left(k_{x}\right) \cos \left(k_{y}\right),
\end{gathered}
$$

where $t$ is the electron jump energy between the nearest atoms in the lattice and $t^{\prime}$ is the energy of the jump to the next nearest atoms. In the study, all results were given in the unit of the jump integral: $t=1\left(t^{\prime}=0.1 t\right)$. For a triangular lattice $(\mathrm{TR})$, the dispersion relation has the form

$$
\varepsilon_{\boldsymbol{k}}=-2 t\left[\cos \left(k_{x}\right)+2 \cos \left(\frac{1}{2} k_{x}\right) \cos \left(\frac{\sqrt{3}}{2} k_{y}\right)\right] .
$$

However, the given functions do not take into account depairing electron correlations. To do this, we use the formalism of thermodynamic Green's functions of the Zubarev type [9] taking into account the Hamiltonian [10]:

$$
H=\sum_{\boldsymbol{k} \sigma} \varepsilon_{\boldsymbol{k}} c_{\boldsymbol{k} \sigma}^{\dagger} c_{\boldsymbol{k} \sigma}+\frac{U}{2} \sum_{\boldsymbol{k} \boldsymbol{k}^{\prime} \boldsymbol{l}} c_{\boldsymbol{k}+\boldsymbol{l} \uparrow}^{\dagger} c_{\boldsymbol{k} \uparrow} c_{\boldsymbol{k}^{\prime} \downarrow}^{\dagger} c_{\boldsymbol{k}^{\prime}+\boldsymbol{l} \downarrow},
$$

where $c_{\boldsymbol{k} \sigma}\left(c_{\boldsymbol{k} \sigma}^{\dagger}\right)$ are the annihilation (creation) operators of the electronic state with momentum $\boldsymbol{k}$ and spin $\sigma \in\{\uparrow, \downarrow\}$. Simple calculations led us to an effective dispersion relation with electron correlations taken, i.e.,

$$
\varepsilon_{\boldsymbol{k}}^{\mathrm{eff}}=\varepsilon_{\boldsymbol{k}}-\frac{U}{2}\langle n\rangle
$$

where $\langle n\rangle=\frac{2}{N} \sum_{\boldsymbol{k}}\left\langle n_{\boldsymbol{k} \downarrow}\right\rangle$ and $\left\langle n_{\boldsymbol{k} \downarrow}\right\rangle=\left\langle c_{\boldsymbol{k} \downarrow}^{\dagger} c_{\boldsymbol{k} \downarrow}\right\rangle$. We assumed in the calculations that on average we have one electron per lattice node $(\langle n\rangle=1)$, and therefore the chemical potential is zero $(\mu=0)$. Given the explicit electron dispersion relation $\varepsilon_{\boldsymbol{k}}^{\text {eff }}$, we can determine the electron density function of states, which is defined by [11]

$$
\rho(\varepsilon)=\frac{1}{N} \sum_{\boldsymbol{k}} \delta\left(\varepsilon-\varepsilon_{\boldsymbol{k}}^{\mathrm{eff}}\right),
$$

where the $\delta(x)$ symbol represents the Dirac distribution: $\delta(x)=\lim _{a \rightarrow 0} \frac{1}{\pi} \frac{a}{a^{2}+x^{2}}$ [12].

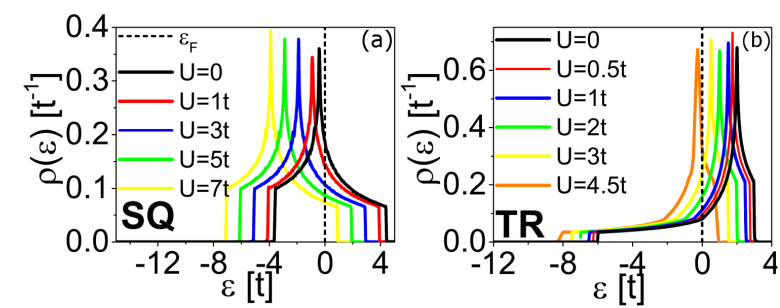

Fig. 1. Electronic density functions of states $\rho(\varepsilon)$ depending on $U$ for a square (a) and a triangular lattice (b). The dashed black line represents the Fermi level.
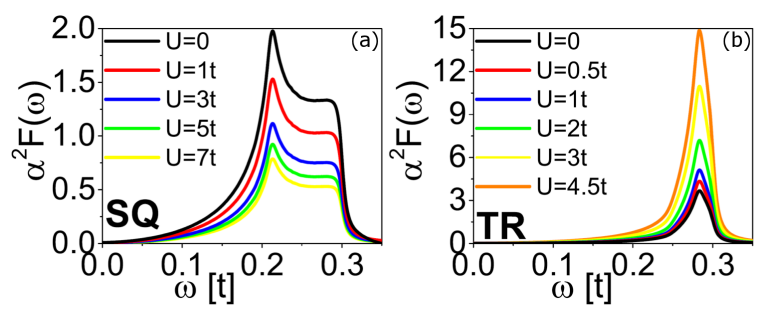

Fig. 2. Eliashberg functions $\alpha^{2} F(\omega)$ depending on $U$ for a square (a) and a triangular lattice (b).

For the electron density of states explicitly dependent on $U$, it is possible to check what influence the electron correlations have on its course. We have shown the results in Fig. 1 for the model systems under consideration. It can be seen that as the $U$ parameter increases, the electron density of state function shifts towards negative energy values in both cases.

In the next stage of the research, we determined the isotropic Eliashberg function given as follows [13]:

$$
\alpha^{2} F(\omega)=\rho(0) \sum_{\boldsymbol{q}} g_{\boldsymbol{q}}^{2} \delta\left(\omega-\omega_{\boldsymbol{q}}\right) .
$$

The symbol $g_{\boldsymbol{q}}$ represents the electron-phonon coupling function defined by $g_{\boldsymbol{q}}=g_{0}|\boldsymbol{q}| \sqrt{\frac{1}{\omega_{\boldsymbol{q}}}}$, where $g_{0}$ is the constant that determines the strength of this interaction. In this study, we assumed $g_{0}=\{0.031,0.03\} \times t^{3 / 2}$, so that the coupling between electrons and phonons was relatively strong for the SQ and TR model $(\lambda=\{2,1.27\})$, respectively. The phonon dispersion relation $\omega_{\boldsymbol{q}}$ was approximated by the harmonic taking into account the vibrations of the nearest atoms and the frequency of the phonon oscillator $\omega_{0}=\{0.15,0.2\} \times t$, respectively for SQ and TR.

We have presented the Eliashberg functions in Fig. 2. It can be seen that with the increasing strength of depairing electron correlations, the superconducting state expires in the square lattice by decreasing values of $\alpha^{2} F(\omega)$. In a triangular lattice, the Eliashberg function assumes higher and higher values with the increase of $U$. This strengthens the superconducting state for this system. This amplification goes up to a certain value of $U$, which is related to the density of states at the Fermi level 

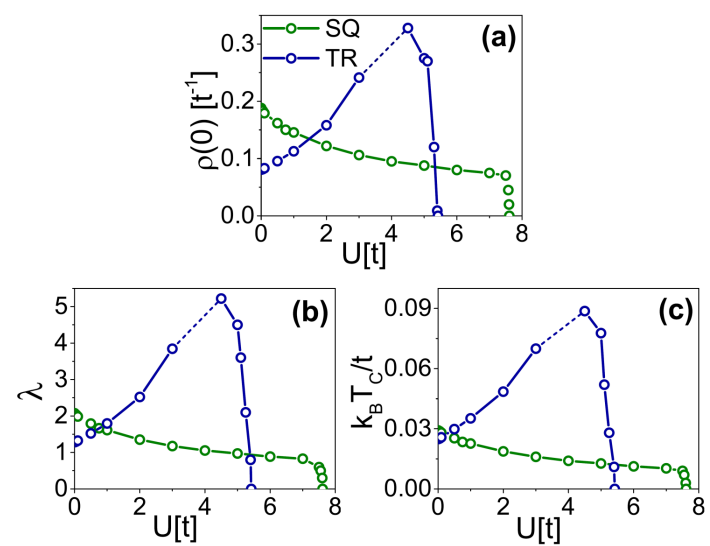

Fig. 3. (a) The density of states value at the Fermi level $\rho(0)$, (b) electron-phonon coupling constant $\lambda$ and (c) critical temperature $T_{c}$ as functions of $U$ for a square (green lines) and a triangular lattice (navy blue lines). The dashed lines indicate the indeterminacy of the results due to the existence of the van Hove singularity in the given range of the $U$ parameter values.

(see Fig. 3a). The superconducting state weakens in the TR model as $\rho(0)$ begins to decline. The value of $U$, for which the superconducting state disappears in both cases, corresponds to the value of the Coulomb integral, when the electron density of state at the Fermi level takes zero values.

The consequence of shifting $\rho(\varepsilon)$ towards negative energy values, with an increase in the $U$ parameter, is a decreasing value of the density of states at the Fermi level $(\rho(0))$ in a square lattice and also its increase for a triangular lattice (see later Fig. 3a). This is due to the fact that the van Hove singularity in the SQ model moves away from the Fermi level with the increase of $U$, and for the TR model the situation is completely opposite. Namely, the electron density of states peak is getting closer and closer to the Fermi level. This has its further consequences - the results for the triangular lattice become undefined (e.g., the dashed lines in Fig. 3) in the vicinity of the van Hove singularity.

Given the explicit Eliashberg function, we can easily calculate the parameters of the superconducting state. We calculated the electron-phonon coupling constant from

$$
\lambda=2 \int_{0}^{\infty} \mathrm{d} \omega \frac{\alpha^{2} F(\omega)}{\omega} .
$$

The critical temperature can be estimated with good accuracy from the Allen-Dynes formula [14]

$$
k_{\mathrm{B}} T_{c}=\frac{\omega_{\mathrm{ln}}}{1.2} f_{1} f_{2} \exp \left(-1.04 \frac{(1+\lambda)}{\lambda}\right),
$$

where $k_{\mathrm{B}}$ is the Boltzmann constant. The logarithmic phonon frequency is determined by

$$
\omega_{\ln }=\exp \left(\frac{2}{\lambda} \int_{0}^{\infty} \mathrm{d} \omega \frac{\alpha^{2} F(\omega)}{\omega} \ln (\omega)\right) .
$$

The symbols $f_{1}$ and $f_{2}$ define the expressions:

$$
f_{1}=\left(1+(\lambda / 2.46)^{\frac{3}{2}}\right)^{\frac{1}{3}}
$$

and

$$
f_{2}=1+\left(\frac{\sqrt{\omega_{2}}}{\omega_{\ln }}-1\right) \frac{\lambda^{2}}{\lambda^{2}+\Lambda_{1}^{2}},
$$

where $\Lambda_{1}=1.82\left(\sqrt{\omega_{2}} / \omega_{\ln }\right)$. The second moment of the normalized weighting function is given by

$$
\sqrt{\omega_{2}}=\sqrt{\frac{2}{\lambda} \int_{0}^{\infty} \mathrm{d} \omega \alpha^{2} F(\omega) \omega} .
$$

The form of the Eliashberg function is influenced by the value $\rho(0)$, which can be seen in (6), so we also analyzed the electron density of state at the Fermi level as a function of $U$.

Let us summarize the results for a square lattice (green lines and symbols) and a triangular lattice (navy blue lines and symbols) in Fig. 3. It can be easily seen that the superconducting state parameters follow a similar course to the electron state density at the Fermi level, which proves that mainly the $\rho(0)$ values have a decisive influence on the superconducting state results.

We obtained critical values of the on-site Hubbard integral in which the superconducting state disappears: $U_{c}=7.61 t$ for a SQ lattice and $U_{c}=5.42 t$ for a TR lattice. The weakening nature of the superconducting state can also be observed, that is, the decreasing $\lambda$ and $T_{c}$ for the entire range of $U$ values in the SQ model. Moreover, Fig. 3 also shows that the maximum value of $\lambda=2.1$ and $k_{\mathrm{B}} T_{c}=0.029 t$ in a square lattice occurs for $U=0$, from which it can be concluded that the increasing force of the repulsion of the electrons destroys the superconducting state in this system. When the value of the jump integral is $t=250 \mathrm{meV}$, the maximum critical temperature equals $T_{c}=84.5 \mathrm{~K}$. In the TR model, we can see both the range $U$ for which the superconducting state will be amplified $U=(0.01,3) t$ and where it disappears $U=(4.5,5.42) t$. The increasing Coulomb interaction strengthens the superconducting state in the low ranges of the $U \in(0.01,3) t$ parameter. Further, it causes that the electron-phonon coupling constant increase to 3.85. The maximum value of $\lambda$ that was achieved in the calculations for the TR model as high as 5.22 was for $U=4.5 t$. This has its consequences - the maximum critical temperature value is $T_{c}=\{202.9,257.2\} \mathrm{K}$ for $U \in\{3,4.5\} t$ and assuming $t=250 \mathrm{meV}$. Typically, the electron hopping force must be strong enough to allow the electron to break away from the atomic nucleus and jump to an adjacent lattice node. Therefore, the value of $t$ also depends on the lattice shape.

In the paper, we assume that the distance between the atoms of the lattice is the same in both cases (the lattice constant is equal to one). Here, the value of $t=250 \mathrm{meV}$ is an example value chosen to illustrate the real values of the parameters 
of the superconducting state in the models under consideration. This value is used in modeling of copper-oxygen planes in cuprates. In these planes, copper and oxygen atoms are arranged in the shape of a square lattice.

\section{Conclusions}

We analyzed the influence of depairing electron correlations on $\rho(\varepsilon)$, which we determined on the basis of the effective dispersion relation taking into account the on-site Coulomb integral $U$, for electrons of a square and triangular lattice. This made it possible to calculate $\alpha^{2} F(\omega)$ for specific values of the Coulomb repulsion force and on its basis to determine the basic parameters of the superconducting state. As a consequence, we found that in the SQ model, the superconducting state disappears throughout the course of $U$, assuming the maximum $\lambda=2.1$ and $T_{c}=84.5 \mathrm{~K}$ for $U=0$ and disappears completely for $U_{c}=7.61 t$. In a triangular lattice, the superconducting state is amplified with increasing Coulomb interaction, reaching $\lambda=3.85$ and $T_{c}=202.9 \mathrm{~K}$ for $U=3 t$. Furthermore, the superconducting state of the triangular system tends to decrease in the range of $U \in(4.5 .5 .42) t$, assuming the maximum value of $\lambda=5.22$ and $T_{c}=257.2 \mathrm{~K}$ for $U=4.5 t$.

\section{References}

[1] G.M. Eliashberg, Sov. Phys. JETP 11, 696 (1960).

[2] J.P. Carbotte, Rev. Mod. Phys. 62, 1027 (1990).

[3] P. Morel, P.W. Anderson, Phys. Rev. 125, 1263 (1962).

[4] J. Bauer, J.E. Han, O. Gunnarsson, Phys. Rev. B 87, 054507 (2013).
[5] J. Bauer, J.E. Han, O. Gunnarsson, J. Phys. Condens. Matter 24, 492202 (2012).

[6] M. Kostrzewa R. Szczęśniak, J.K. Kalaga, I.A. Wrona, Sci. Rep. 8, 11957 (2018).

[7] F. Giustino, Rev. Mod. Phys. 89, 015003 (2017).

[8] E. Kaxiras, Atomic and Electronic Structure of Solids, CUP, New York 2003.

[9] W. Gasser, E. Heiner, K. Elk, Greensche Funktionen in Festkörper und Vielteilchenphysik, VILEY-VCH Verlag, Weinheim 1999 (in German).

[10] J. Hubbard, Proc. Roy. Soc. A 276, 238 (1963).

[11] W.A. Harrison, Electronics structure and the properties of solids, Dover Publications, Michigan 1989.

[12] G.B. Arfken, H.J. Weber, Mathematical Methods for Physicists, AP Boston, Massachusetts 2000.

[13] W.L. McMillan, Phys. Rev. 167, 331 (1968).

[14] P.B. Allen, R.C. Dynes, Phys. Rev. B 12, 905 (1975). 